\title{
Compostos bioativos e o controle de carrapatos em bovinos
}

\section{Carlos Frederico de Carvalho Rodrigues, João Elzeário Castelo Branco Iapichini*, Daniela Pontes Chiebao, Fábio Henrique de Lima Gabriel}

Pesquisadores Científicos da APTA - Agencia Paulista de Tecnologia dos Agronegócios / Secretaria de Agricultura e Abastecimento do Estado de São Paulo

*Autor para correspondência, E-mail: iapichini@gmail.com

RESUMO. Frente ao histórico fenômeno da resistência dos parasitas, em especial do Rhipicephalus (Boophilus) microplus, aos diversos princípios ativos sintéticos disponíveis no mercado, constantes investigações na busca de novas moléculas eficazes no controle de ectoparasitas são impulsionadas, preferencialmente por moléculas bioativas naturais. $\mathrm{O}$ uso tradicional e empírico de diversas fontes de compostos bioativos pelos produtores rurais e outros não os credenciam como alternativa para o controle de carrapatos. Desta forma, é necessária a comprovação científica da eficácia desses compostos, e assim diversos fitoterápicos têm sido testados para controle do carrapato dos bovinos. O efetivo controle do carrapato Rhipicephalus (Boophilus) microplus através da validação e difusão dos princípios da associação das diferentes estratégias (controle químico, controle biológico, prospecção e validação de compostos bioativos, seleção e descarte de animais resistentes e susceptíveis) aliadas aos conhecimentos do ciclo biológico do parasita contribuirá para a mitigação dos prejuízos diretos e indiretos causados pelas infestações do carrapato na cadeia de produção de bovinos no Brasil, e, por conseguinte em diversos aspectos da saúde pública, em especial do consumidor final.

Palavras chave: Bovino, carrapato, controle

\section{The bioactive compounds and cattle tick control}

ABSTRACT. Opposite the historic parasite resistance phenomenon, especially the Rhipicephalus (Boophilus) microplus, the various synthetic active ingredients available in the market, constant investigations in the search for new molecules effective in controlling ectoparasites are driven, preferably by natural bioactive molecules. The traditional and empirical use of different sources of bioactive compounds by farmers and others not qualify it as an alternative for the control of ticks. Thus, scientific evidence of the efficacy of such compounds, and so many natural products has been tested for the control of cattle tick is required. Effective control of Rhipicephalus (Boophilus) microplus through validation and dissemination of association principles of different strategies (chemical control, biological control, exploration and validation of bioactive compounds, selection and disposal of resistant and susceptible animals) combined with the knowledge of the cycle biological parasite contribute to the mitigation of direct and indirect damage caused by tick infestations in cattle production chain in Brazil, and therefore in many aspects of public health, in particular the final consumer.

Keywords: Cattle, tick, control

\section{Introdução}

O carrapato Rhipicephalus (Boophilus) microplus, importante hospedeiro e transmissor de doenças de bovinos, exige para seu controle investimentos em instalações, insumos, equipamentos e mão de obra. Infestações reduzem o desempenho do rebanho e elevam os custos de produção, sendo assim responsável por prejuízos anuais de mais de US\$ 2 bilhões (Grisi et al., 2002), além do risco de contaminação ambiental.

Frente ao histórico fenômeno da resistência dos parasitas, em especial do Rhipicephalus (Boophilus) microplus, aos diversos princípios 
ativos sintéticos disponíveis no mercado, constantes investigações na busca de novas moléculas eficazes no controle de ectoparasitas são impulsionadas, preferencialmente por moléculas bioativas naturais.

Diversos estudos contemporâneos avaliam as potencias ações antiparasitárias de diferentes fitoterápicos através de testes in vitro e in vivo, em especial aqueles voltados para o controle de ectoparasitas, com destaque para os carrapatos e outros ácaros (Borges et al., 2011).

Quando comparado aos produtos sintéticos, a utilização de compostos fitoquímicos no controle de parasitas apresentam diversas vantagens: rapidamente degradáveis; obtidos a partir de recursos renováveis; fenômeno da resistência a essas substâncias é um processo lento; não deixam resíduos tóxicos nos alimentos e fácil acesso e obtenção pelos pecuaristas, o que representa menor custo de produção (Roel, 2001).

Além disso, os compostos bioativos devem atender os critérios de eficácia; de toxidade mínima ou inexistente para animais silvestres, domésticos e pessoas; de bom poder residual; economicamente viável; de fácil manuseio e com pequeno período de carência para consumo de carnes e leite (Borges et al., 2011).

\section{Prospecção de compostos bioativos}

O uso tradicional e empírico de diversas fontes de compostos bioativos pelos produtores rurais e outros não os credenciam como alternativa para o controle de carrapatos. Desta forma, é necessária a comprovação científica da eficácia desses compostos, e assim diversos fitoterápicos têm sido testados para controle do carrapato dos bovinos.

Sutherst et al., (1982) atribuíram a uma mistura de $\alpha$-pineno e $\beta$-pineno, presentes nas leguminosas tropicais Stylosanthes scabra e $S$. viscosa, o efeito tóxico que provocaram a imobilização e morte de $100 \%$ das larvas de carrapato após 24 horas de contato com essas leguminosas.

A composição química do óleo essencial do capim-gordura (Melinis minutiflora Beauv.), e sua atividade carrapaticida e dos terpenos $\alpha$ pineno e $\beta$-pineno foi estudada por Prates et al.(1993), causando $100 \%$ de letalidade das larvas do R.(B.) microplus em 10 minutos. A análise do óleo do capim-gordura não identificou os monoterpenos $\alpha$-pineno e $\beta$-pineno como um dos seus componentes.

Segundo Chagas et al. (2002) a eficácia máxima $(100 \%)$ sobre fêmeas ingurgitadas foi obtida pelo óleo de Eucaliptus citriodora, quando a concentração de $25 \%$ foi testada, e pelos óleos de $E$. globulus e E. staigeriana, nas respectivas concentrações de $10 \%$ e $15 \%$. + emulsificantes.

A eficiência do extrato etanolico de Dahlstedtia pentaphylla (timbó) sobre teleóginas de $R$. microplus de uma cepa do Vale do ParaibaSP, variou de $13,49 \%$ a $98,68 \%$, de acordo com as diluições testadas (Pereira \& Famadas, 2004).

Diferentes concentrações do extrato alcoólico de Cymbopogon citratus (capim-cidreira) determinaram certo controle do carrapato dos bovinos, variando de $52,22 \%$ a $55,31 \%$ (Heimerdinger, 2005). Pires (2006) avaliou efeito do extrato aquoso e etanólico da planta Simarouba versicolor, St. Hill (Pau-Paraíba) sobre a atividade reprodutiva "in vitro" dos carrapatos R.(B.) microplus, concluindo que os extratos aquosos e etanólicos do pau-paraíba inibiram em $100 \%$ a ovipostura e eclosão dos ovos de teleóginas imersas entre 10 a 60 minutos nos extratos testados e que ambos provocaram a morte das larvas após seis horas de contato. Assim, podem ser considerados com alternativa de controle. Silva Filho (2007) avaliou in vitro a ação antiparasitária do extrato aquoso e etanólico do angico preto (Anadenanthera macrocarpa) (Benth.) Brenan sobre o carrapato R.(B.) microplus, concluindo que os extratos apresentaram efeito larvicida, embora o extrato etanólico tenha sido mais eficiente e, portanto, potencial alternativa no controle desse ectoparasito. Já o óleo de Carapa guianensis (andiroba) obteve eficácia de $100 \%$ sobre teleóginas do $R$. microplus em todas as diluições testadas (Farias et al., 2007). Os extratos hexânicos de frutos verdes de Melia azedarach (Santa Barbara ou cinamomo) sobre fêmeas ingurgitadas apresentou crescentes elevações de eficácia até $100 \%$, conforme aumento das concentrações (Sousa et al., 2008). Variações de eficácia do óleo de Cymbopogon nardus (citronela) quanto à ação acaricida foram registradas entre $0,71 \%$ e $92,1 \%$, de acordo com as diferentes concentrações avaliadas. (Olivo et al., 2008). Agnolin et al. (2010) consideraram a média de eficácia no controle do carrapato de 93,$8 ; 22,5$ e $39,1 \%$ para o amitraz a $0,025 \%$ e óleo de citronela a 3 e $4 \%$, respectivamente. 
Os testes realizados in vitro sobre larvas de $B$. microplus com fitoterápicos alcoólicos de eucalipto (Eucalyptus sp. L.), açoita-cavalo (Luchea speciosa L.), sucupira (Bowdichia virgiliodes L.), andiroba (Carapa guianensis Aubl.) e romã (Punica granatum L.) a $10 \%$ apresentaram $100 \%$ de mortalidade larval de $B$. microplus em 10, 20 e 40 minutos de imersão, enquanto o nim (Azadirachta indica) obteve $35,9 \% ; 54,3 \%$ e $70,8 \%$ de mortalidade, respectivamente, verificando-se sua ineficácia (Neves et al., 2009).

Broglio-Micheletti et al. (2009) avaliaram extratos orgânicos alcoólicos 2\% (peso/volume) de sementes de Annona muricata L. (Annonaceae) (graviola); flores de Syzygium malaccensis (L.) (Myrtaceae) (jambo); folhas de Cymbopogon citratus (DC.) Stapf (Poaceae) (capim-santo); folhas de Azadirachta indica A. Juss (Meliaceae) (nim); e extrato hexanico na mesma concentração de $A$. indica (sementes). $\mathrm{O}$ extrato de A. muricata apresentou o maior poder acaricida, com eficácia de $100 \%$, seguido dos extratos de $S$. malaccensis (75 e 59,24\%) e $A$. indica (65 e 38,49\%). Houve $100 \%$ de redução na eclosão das larvas quando se utilizou o extrato de sementes de A. muricata.

Bogni et al. (2010) avaliaram os terpenos farnesol, nerol, nerolidol, geraniol, d-pineno, carvona, ácido gerânico e isopulegol e duas cumarinas sintéticas $(\mathrm{C} 1$ e $\mathrm{C} 2)$ quanto à sua atividade sobre fêmeas adultas e larvas de Rhipicephalus (Boophilus) microplus. Esses autores concluem que os terpenos isoladamente não apresentaram resultados muito significativos, mas acredita-se que sua associação poderia potencializar a ação carrapaticida. Entretanto, esta estratégia poderia dificultar a elaboração de uma formulação futura, em termos de controle de qualidade e praticidade.

A obtenção de eficácia parcial $(\mathrm{EP}=50,0 \%)$ do extrato do pinheiro brasileiro (Araucária) sobre as fêmeas ingurgitadas de $R$. microplus indica seu potencial acaricida (Castro et al.2009),o que direciona a novos experimentos com os extratos de outras pináceas como fonte de compostos fenólicos bioativos, como aqueles encontrados no Pinus elliottii e no Pinus taeda (Dutra et al., 2010). A terebintina é uma mistura de isômeros monoterpênicos bicíclicos (predominantemente Alfa Pineno e Beta Pineno), obtida pelo fracionamento/destilação e condensação da goma-resina de pinheiros (Pinus elliottii e Pinus taeda). Rodrigues at al. (2013) verificaram ação deletéria da terebintina associada à lecitina de soja (formulação pour on a $10 \%$ ) sobre ninfas e teleóginas dos poucos carrapatos presentes nos animais experimentais avaliados para controle de bernes e moscas dos chifres. Veríssimo et al. (2013) estudaram o efeito do terpeno D-limoneno e do óleo essencial de laranja e uma mistura dos dois (40\% terpeno limoneno e $20 \%$ óleo de laranja), sobre a relação do peso de ovos e peso das teleóginas de carrapatos dos bovinos, havendo diferença significativa entre tratamentos, porém, nenhum diferiu do tratamento testemunha (água destilada).

Costa et al., (2014) relataram significativa eliminação de $70 \%$ de carrapatos em bovinos leiteiros após aplicação de manipueira (resíduo líquido rico em ácido cianídrico, obtido da produção de farinha de mandioca) associada ou não ao nim (azadiractina), enquanto que nos animais tratados apenas com nim esse não foi efetivo.

\section{Considerações finais}

Discorrendo sobre as perspectivas para o uso de extratos de plantas para o controle do carrapato de bovinos Rhipicephalus (Boophilus) microplus, Borges et al. (2011) atentam que estudos in vitro de extratos de plantas têm apresentado resultados promissores, mas a maioria destes extratos não têm sido testada em animais para validar estes resultados. Esses mesmos autores consideram que dificuldades para preparar formulações apropriadas, diferenças na composição química de plantas de uma mesma espécie devido a fatores intrínsecos e extrínsecos e informações esparsas sobre os princípios ativos são entraves que precisam ser solucionados visando o desenvolvimento deste campo de pesquisa.

Outro entrave a ser observado no uso de moléculas bioativas alternativas é a dificuldade de produção da matéria-prima em escala industrial a custos competitivos comparados aos produtos sintéticos comerciais.

Como estratégia de controle do carrapato Rhipicephalus (Boophilus) microplus no Brasil, Veríssimo (2013) considera a criação de bovinos resistentes, com destaque para as raças zebuínas e seus cruzamentos, como a forma mais eficaz (população de carrapatos), econômica (sem insumos) e ecológica (sem resíduos) de controle biológico do carrapato de bovinos. 
Vislumbra-se o efetivo controle do carrapato Rhipicephalus (Boophilus) microplus através da validação e difusão dos princípios da associação das diferentes estratégias (controle químico, controle biológico, prospecção e validação de compostos bioativos, seleção e descarte de animais resistentes e susceptíveis) aliadas aos conhecimentos do ciclo biológico do parasita.

Assim, a mitigação dos prejuízos diretos e indiretos causados pelas infestações do carrapato Rhipicephalus (Boophilus) microplus no rebanho bovino brasileiro irá refletir significativamente no aumento da produtividade desses sistemas de produção animal, contribuindo de maneira diferenciada na sua sustentabilidade, e, por conseguinte em diversos aspectos da saúde pública, em especial do consumidor final.

\section{Referências Bibliográficas}

Bogni, S., Chagas, A.C.S. et al. 2010. Ação acaricida de terpenos sobre o carrapato bovino rhipicephalus (boophilus) microplus. In: Congresso Brasileiro De Parasitologia Veterinária, 16., ampo Grande. Anais/CDROM.campo Grande: CBPV.

Borges, L.M.F., Sousa, L.A.D. \& Barbosa, C.S. 2011. Perspectives for the use of plant extracts to control the cattle tick Rhipicephalus (Boophilus) microplus. Revista Brasileira Parasitologia e Veterinária, 20, 89-96.

Broglio-Micheletti, S.M.F., Valente, E.C.N., Souza, L.A., Dias, N.S. \& Araújo, A.M.N. 2009. Extratos de plantas no controle de Rhipicephalus (Boophilus) microplus (Canestrini, 1887) (Acari: Ixodidae) em laboratório. Revista Brasileira Parasitologia e Veterinária, 18, 44-48.

Castro, K.N.C. et al. 2009. Avaliação in vitro do extrato do pinheiro brasileiro para controle do carrapato dos bovinos. Revista Brasileira De Agroecologia, 4, 2575-2577.

Costa, C. Silva; Melo, T.L. . \& Pires, C.R.S. 2014. Uso e aplicações do nim e manipueira como métodos alternativos para combate de carrapatos em bovinos leiteiros. In: Congresso Brasileiro de Medicina Veterinária, 40, 2013, Salvador. Anais. Revista de Educação Continuada em Medicina Veterinária $e$ Zootecnia, 12, 65.

Chagas, A.C.S. et al. 2002. Efeito acaricida de óleos essenciais e concentrados emulsionaveis de Eucalyptus spp em Boophilus microplus.
Brazilian Journal of Veterinary Research and Animal Science, Sao Paulo, 39, 247-253.

Dutra, B.K; Oliveira, M.R., Braghirolli, F.M., Paradeda, N.L., Villavicencio, B., Razzera, B.N., Failace, D.M., Pöerschke, F., Fernandes, F.A., Astarita, L. \& Oliveira, G.T. 2010. Avaliação do impacto no ambiente de metabólitos produzidos por pinus taeda (coniferae) utilizando indicadores biológicos. In: Salão de Iniciação Científica, 11, 2010, Porto Alegre. Proceedings. Porto Alegre: PUCRS.

Farias, M.P.O. et al. 2007. Eficacia in vitro do oleo de Carapa guianensis Aubl. (andiroba) no controle de Boophilus microplus (Acari: Ixodidae). Revista Brasileira de Plantas Medicinais, Botucatu, 9, 69-71,

Grisi L., Massard C.L., Moya Borja G.E. \& Pereira J.B. 2002. Impacto econômico das principais ectoparasitoses em bovinos no Brasil. A Hora Veterinária, 21 (125), 8-10.

Heimerdinger, A. 2005. Extrato alcoólico de capim-cidreira (Cymbopogon citratus) no controle do carrapato (Boophilus microplus) de bovinos leiteiros. 2005. 75 f. Dissertacao (Mestrado em Zootecnia) - Universidade Federal de Santa Maria, Santa Maria.

Olivo, C.J. et al. 2008. Óleo de citronela no controle do carrapato de bovinos. Ciência Rural, Santa Maria, 38, 406-410.

Neves, A.P. et al., 2009. Efeito do extrato hidroalcoólico de Capim-limão (Cympobogon citratus) sobre o ciclo do carrapato Rhipicephalus microplus em novilhas leiteiras Revista Brasileira de Agroecologia, 4, 401 404.

Pereira, J R., Famadas, K.M. 2004. Avaliação "in vitro" do extrato da raiz do timbó (Dahlstedtia pentaphylla) (leguminosae, Papilionoidae, Millettieda) sobre Boophilus microplus (Canestrini,1887) na região do Vale do Paraíba, São Paulo, Brasil. Arquivos do Instituto Biológico, São Paulo, 71, 443-50.

Pires, J.E.P. 2006. Efeito dos extratos aquoso e etanólico de planta Simarouba versicolor St. Hill "in vitro" sobre larvas e teleóginas de carrapatos Boophilus microplus Canestrini, 1887 e Rhipicephalus sanguineus Latreille, 1806, 2006. 49 f. Dissertação (Mestrado em Ciência Animal) - Universidade Federal do Piauí. 
Prates, H.T. et al. 1993. Atividade carrapaticida e composição química do óleo essencial do capim-gordura. Pesquisa Agropecuária Brasileira, 28, 621-625.

Rodrigues, C.F.C., Iapichini, J.E.C.B., Freire Neto, A.O.L., Veríssimo, C.J., Katiki, L.M., Freire, L.V. \& Santiago, C.M. 2013. Potencial da goma-resina de Pinus elliottii no controle de ectoparasitas de bovinos. In: Sboe, Simpósio Brasileiro De Óleos Essenciais, 7., Pará. Anais... UFOPA: Santarém, PA.

Roel, A.R. 2001. Utilização de plantas com propriedades inseticidas: uma contribuição para o Desenvolvimento Rural Sustentável. Revista Internacional de Desenvolvimento Local. 1, 43-50

Sousa, L.A.D. et al. 2008. Avaliação da eficácia de extratos oleosos de frutos verdes e maduros de cinamomo (Melia azedarach) sobre Rhipicephalus (Boophilus) microplus (Acari: Ixodidae). Revista Brasileira de Parasitologia Veterinária, São Paulo, 17, 36-40.

Silva Filho, M.L. 2007. Avaliação in vitro da ação antiparasitária do extrato aquoso e etanólico do angico preto (Anadenanthera macrocarpa ) (Benth.) Brenan sobre o carrapato Rhipicephalus (Boophilus) microplus ( Canestrini, 1887 ). 2007. 50 f.
Dissertação (Mestrado em Ciência Animal) Universidade Federal do Piauí.

Sutherst, R.W., Jones, R.J. \& Schnitzerling, H.J. 1982. Tropical legumes of the genus Stlylosanthes immobilize and kill cattle ticks. Nature, 295, 320-321.

Veríssimo, C.J. 2013. Controle biológico do carrapato do boi, Rhipicephalus (Boophilus) microplus, no Brasil. Revista de Educação Continuada em Medicina Veterinária e Zootecnia do CRMV-SP, 11, 14-23.

Veríssimo, C.J. et al. 2013. Efeito de óleo essencial de laranja, do terpeno D-limoneno e mistura (óleo de laranja e D-limoneno) sobre o carrapato-do-boi. In: 26a. RAIB - Reunião Anual do Instituto Biológico, 2013, São Paulo. O Biológico. São Paulo: Instituto Biológico, 75, 75-75.

Recebido em Março 24, 2015

Aceito em Junho 9, 2015

License information: This is an open-access article distributed under the terms of the Creative Commons Attribution License, which permits unrestricted use, distribution, and reproduction in any medium, provided the original work is properly cited. 